EESTI NSV TEADUSTE AKADEEMIA TOIMETISED. XIII KÖIDE FOOSIKA-MATEMAATIKA- JA TEHNIKATEADUSTE SEERIA. 1964, NR. 3

ИЗВЕСТИЯ АКАДЕМИИ НАУК ЭСТОНСҚОЙ ССР. ТОМ ХІІІ СЕРИЯ ФИЗИКО-МАТЕМАТИЧЕСКИХ И ТЕХНИЧЕСКИХ НАУК. 1964, N3 3

\title{
ПРИНЦИП МАЖОРАНТ И МЕТОД ХОРД
}

\author{
с. УЛьм,
}

кандидат физико-математических наук

А. С. Сергеев $\left[{ }^{3}\right]$ и И. В. Шмидт $\left[{ }^{5,}{ }^{6}\right]$ обобщили (метод хорд для приближенного решения нелинейных уравнений в линейных нормированных пространствах. Ими доказаны теоремы, дающие достаточные условия для сходимости метода хорд к решению уравнения. В данной статье мы используем для исследования сходимости метода хорд принцип мажорант, успешно примененный Л. В. Канторовичем [1] для метода Ньютона. При этом из доказанных ниже теорем вытекают как частные случаи результаты А. С. Сергеева и Н. В. Шмидта в уточненном виде. Отметим еще, что некоторые соображения о применении принципа мажорант для исследования сходимости метода хорд были опубликованы автором ранее (см. [ $\left.\left.{ }^{4}\right]\right)$.

Пусть $P(x)$ - нелинейный оператор, переводящий линейное нормированное пространство $X$ в пространство $\mathcal{Y}$ того же типа.

Оп ределени е 1. Оператор $P\left(x^{\prime}, x^{\prime \prime}\right)$ называется аналогом разделенных разностей первого порядка оператора $P(x)$, если

$1^{\circ}$ для каждого фиксированного $x^{\prime}, x^{\prime \prime} \in X$ оператор $P\left(x^{\prime}, x^{\prime \prime}\right) \in(X \rightarrow \boldsymbol{y})$, т. е. является линейным оператором;

$2^{\circ} P\left(x^{\prime}, x^{\prime \prime}\right)\left(x^{\prime}-x^{\prime \prime}\right)=P\left(x^{\prime}\right)-P\left(x^{\prime \prime}\right)$

$3^{\circ} P(x, x)=P^{\prime}(x)$, где $P^{\prime}(x)$ производная оператора $P(x)$ в смысле Гато.

Отметим, что от своих аргументов $x^{\prime}, x^{\prime \prime} \in X$ оператор $P\left(x^{\prime}, x^{\prime \prime}\right)$ 3aвисит в общем нелинейно.

Для приближенного решения уравнения

$$
P(x)=0
$$

рассмотрим итерационные методы

K

$$
\begin{aligned}
& x_{n+1}=x_{n}-\Lambda_{1} P\left(x_{n}\right), \\
& x_{n+1}=x_{n}-\Lambda_{n} P\left(x_{n}\right),
\end{aligned}
$$

где $\Lambda_{n}=\left[P\left(x_{n}, x_{n-1}\right)\right]^{-1} ; n=1,2,3, \ldots ; x_{0}, x_{1}$ - два различных начальных приближения к решению $x^{*}$ уравнения (1). Методы (2) и (3) являются соответственно модифицированным и основным методами хорд.

Вместе с уравнением (1) рассмотрим вещественное мажорантное уравнение

$$
Q(t)=0
$$


и для его решения методы

$$
\begin{gathered}
t_{n+1}=t_{n}+Q\left(t_{n}\right), \\
t_{n+1}=t_{n}-\frac{Q\left(t_{n}\right)}{Q\left(t_{n}, t_{n-1}\right)},
\end{gathered}
$$

где $n=1,2, \ldots ; t_{0}, t_{1}$ - начальные приближения к решению $t^{*}$ уравнения (4); $t_{0}<t_{1}=0$.

В дальнейшем предположим существование в интересуюших нас областях разделенных разностей первого порядка оператора $P$ и функции $Q$. Предположим, что $P$ (соответственно $Q$ ) является непрерывным в окрестности $x^{*}$ (соответственно $\left.t^{*}\right)$. Через $E$ обозначим тождественный оператор банахового пространства $X$. Через $S[\bar{x}, r]$ обозначим закрытую сферу $\|x-x\| \leqslant r$ пространства $X$.

Вначале докажем некоторые теоремы о сходимости метода (2).

Tеорема 1. Пусть

$1^{\circ}\left\|x_{1}-x_{0}\right\| \leqslant-t_{0}$;

$2^{\circ}$ существует $\Lambda_{\mathrm{i}}=\left[P\left(x_{1}, x_{0}\right)\right]^{-1}$, причем $\left\|\Lambda_{1} P\left(x_{1}\right)\right\| \leqslant Q(0)$; $3^{\circ}\left\|E-\Lambda_{1} P\left(x^{\prime}, x^{\prime \prime}\right)\right\| \leqslant 1+Q\left(t^{\prime}, t^{\prime \prime}\right)$, если $x^{\prime}, x^{\prime \prime} \in S\left[x_{1}, t^{*}\right] u$

$$
\left\|x^{\prime}-x^{\prime \prime}\right\| \leqslant t^{\prime}-t^{\prime \prime},\left\|x^{\prime}-x_{1}\right\| \leqslant t^{\prime},\left\|x^{\prime \prime}-x_{1}\right\| \leqslant t^{\prime \prime} ; t^{\prime}, t^{\prime \prime} \leqslant t^{*} \text {; }
$$

$4^{\circ}$ уравнение (4) имеет положительное решение.

Тогда уравнение (1) имеет в сфере $S\left[x_{1}, t^{*}\right]$ решение $x^{*}$, $\kappa$ которому сходится последовательность (2) со скоростью

$$
\left\|x^{*}-x_{n}\right\|_{1} \leqslant t^{*}-t_{n} \quad(n=0,1, \ldots),
$$

где $t^{*}$ - наименьший положительный корень уравнения (4);

$t_{n}$ - n-ое приближение по методу (5) $\kappa t^{*}$.

Д ока зательство. Прежде всего покажем, что в условиях теоремы последовательность (5) сходится к $t^{*}$. Используем принцип полной индукции. Допустим, что

$$
0=t_{1}<t_{2} \leqslant \ldots \leqslant t_{n} \leqslant t^{*}
$$

и покажем, что $t_{n} \leqslant t_{n+1} \leqslant t^{*}$.

По формуле (5) $t_{n+1}-t_{n}=Q\left(t_{n}\right) \geqslant 0$, так как $Q(0)=Q\left(t_{1}\right)>0^{*}$ и $t^{*}$ - наименьшее положительное решение уравнения (4). Дальше получим

$$
\begin{aligned}
t^{*}-t_{n+1}=t^{*} & -t_{n}-Q\left(t_{n}\right)=t^{*}-t_{n}+\left[Q\left(t^{*}\right)-Q\left(t_{n}\right)\right]= \\
& =\left[1+Q\left(t^{*}, t_{n}\right)\right]\left(t^{*}-t_{n}\right) \geqslant 0,
\end{aligned}
$$

так как по условию $3^{\circ} 1+Q\left(t^{*}, t_{n}\right) \geqslant 0$. Последовательность $\left\{t_{n}\right\}$ яв. ляется монотонно возрастающей и ограниченной. Итак, существует $\lim _{n \rightarrow \infty} t_{n}=\bar{t}$. Переходя к пределу в (5) $(n \rightarrow \infty)$, получим, что $Q(\bar{t})=0$, т. e. $\bar{t}=t^{*}$.

Из условия $2^{\circ}$ вытекает, что $\left\|x_{2}-x_{1}\right\|=\left\|\Lambda_{1} P\left(x_{1}\right)\right\| \leqslant Q(0)=$ $=t_{2}-t_{1}$. Используем опять принцип полной индукции. Допустив, что $\left\|x_{k}-x_{k-1}\right\| \leqslant t_{k}-t_{k-1}(k \leqslant n)$, покажем, что $\left\|x_{n+1}-x_{n}\right\| \leqslant t_{n+1}-t_{n}$.

* Если $Q\left(t_{1}\right)=0$, то начальное прнближение $x_{1}$ является решением уравнення (1). 
На основании формулы (2) и определения 1

$$
\begin{gathered}
\left\|x_{n+1}-x_{n}\right\|=\left\|\Lambda_{1} P\left(x_{n}\right)\right\|= \\
=\| \Lambda_{1}\left\{P\left(x_{n-1}\right)+P\left(x_{1}, x_{0}\right)\left(x_{n}-x_{n-1}\right)+\right. \\
\left.+\left[P\left(x_{n}, x_{n-1}\right)-P\left(x_{1}, x_{0}\right)\right]\left(x_{n}-x_{n-1}\right)\right\} \|= \\
=\left\|\Lambda_{1}\left[P\left(x_{n}, x_{n-1}\right)-P\left(x_{1}, x_{0}\right)\right]\left(x_{n}-x_{n-1}\right)\right\| \leqslant \\
\leqslant\left\|E-\Lambda_{1} P\left(x_{n}, x_{n-1}\right)\right\|\left\|x_{n}-x_{n-1}\right\| .
\end{gathered}
$$

С. другой стороны

$$
\begin{aligned}
t_{n+1}-t_{n}=Q\left(t_{n}\right)=Q\left(t_{n-1}\right)+Q\left(t_{n}, t_{n-1}\right)\left(t_{n}-t_{n-1}\right)= \\
=t_{n}-t_{n-1}+Q\left(t_{n}, t_{n-1}\right)\left(t_{n}-t_{n-1}\right)= \\
=\left[1+Q\left(t_{n}, t_{n-1}\right)\right]\left(t_{n}-t_{n-1}\right) .
\end{aligned}
$$

По условию $3^{\circ} \quad\left\|E-\Lambda_{1} P\left(x_{n}, x_{n-1}\right)\right\| \leqslant 1+Q\left(t_{n}, t_{n-1}\right)$, так как $\left\|x_{n}-x_{n-1}\right\| \leqslant t_{n}-t_{n-1}$ и очевидным образом $\left\|x_{n}-x_{1}\right\| \leqslant t_{n} \leqslant t^{*}$, $\left\|x_{n-1}-x_{1}\right\| \leqslant t_{n-1} \leqslant t^{*}$. Значит, $\left\|x_{n+1}-x_{n}\right\| \leqslant t_{n+1}-t_{n}$. Отсюда сразу вытекают оценки: $\left\|x_{n+p}-x_{n}\right\| \leqslant t_{n+p}-t_{n}$. Переходя здесь к пределу $(p \rightarrow \infty)$, заключаем существование $x^{*}$ и оценки $\left\|x^{*}-x_{n}\right\| \leqslant t^{*}-t_{n}$. Переходя к пределу $(n \rightarrow \infty)$ в формуле $(2)$, убеждаемся, что $x^{*}$ является решением уравнения (1).

Теорема доказана.

Взяв в качестве мажорантного уравнения (4) уравнение

$$
Q(t) \equiv K t^{2}-(1-K \delta) t+\eta=0,
$$

получим, что справедлива (как следствие из теоремы 1)

Теорема 2. Пусть

$1^{\circ}\left\|x_{1}-x_{0}\right\| \leqslant \delta$;

$2^{\circ}\left\|\Lambda_{1} P\left(x_{1}\right)\right\| \leqslant \eta$;

$3^{\circ}$ для каждого $x^{\prime}, x^{\prime \prime} \in S\left[x_{1}, t^{*}\right]$ справедливо

$\left\|E-\Lambda_{1} P\left(x^{\prime}, x^{\prime \prime}\right)\right\| \leqslant K\left(\left\|x^{\prime}-x_{1}\right\|+\left\|x^{\prime \prime}-x_{0}\right\|\right)$;

$4^{\circ} 2 \sqrt{K \eta}+K \delta \leqslant 1$.

Тогда уравнение (1) имеет в сфере $S\left[x_{1}, t^{*}\right]$ решение $x^{*}, \kappa$ которому последовательность (2) сходится. Справедливы оценки: $\left\|x^{*}-x_{n}\right\| \leqslant$ $\leqslant t^{*}-t_{n}(n=0,1, \ldots)$, гдe

$$
t^{*}=(2 K)^{-1}\left[1-K \delta-\sqrt{\left.(1-K \delta)^{2}-4 K \eta\right]}\right. \text {; }
$$

$t_{n}$ - n-ое приближение по методу (5) $\kappa$ решению $t^{*}$ уравнения (9); $t_{0}=-\delta ; t_{1}=0$.

Д оказательство. Нетрудно проверить, что все условия теоремы 1 выполнены. Неравенство $2 \sqrt{K \eta}+K \delta \leqslant 1$ гарантирует существование положительного решения уравнения (9). Так как $Q(0)=\eta$, то выполнено и условие $2^{\circ}$ теоремы 1 . Если $x^{\prime}, x^{\prime \prime} \in S\left[x_{1}, t^{*}\right],\left\|x^{\prime}-x_{1}\right\| \leqslant t^{\prime}$ и $\left\|x^{\prime \prime}-x_{1}\right\| \leqslant t^{\prime \prime}$, тогда $\left\|E-\Lambda_{i} P\left(x^{\prime}, x^{\prime \prime}\right)\right\| \leqslant K\left(\left\|x^{\prime}-x_{1}\right\|+\left\|x^{\prime \prime}-x_{0}\right\|\right) \leqslant$ $\leqslant K\left(t^{\prime}+t^{\prime \prime}+\delta\right)=1+Q\left(t^{\prime}, t^{\prime \prime}\right)$, т. е. выполнено и условие $3^{\circ}$ теоремы 1 . Выполнение условия $1^{\circ}$ теоремы 1 очевидно. 
О п р едел ени е 2 . Оператор $P\left(x^{\prime}, x^{\prime \prime}, x^{\prime \prime \prime}\right)$ называется аналогом разделенных разностей второго порядка оператора $P(x)$, если

$1^{\circ}$ для каждого фиксированного $x^{\prime}, x^{\prime \prime}, x^{\prime \prime \prime} \in X$ оператор $P\left(x^{\prime}, x^{\prime \prime}, x^{\prime \prime \prime}\right)$ является билинейным оператором из пространства $X$ в пространство $Y$. $2^{\circ} P\left(x^{\prime}, x^{\prime \prime}, x^{\prime \prime \prime}\right)\left(x^{\prime}-x^{\prime \prime \prime}\right)=P\left(x^{\prime}, x^{\prime \prime}\right)-P\left(x^{\prime \prime}, x^{\prime \prime \prime}\right)$.

$3^{\circ} P(x, x, x)=\frac{1}{2} P^{\prime \prime}(x)$, где $P^{\prime \prime}(x)$ - вторая производная оператора $P(x)$ в смысле Гато.

3 а м е ч ани е 1. Если существует $P\left(x^{\prime}, x^{\prime \prime}, x^{\prime \prime \prime}\right)$, то. условие $3^{\circ}$ в теореме 2 можно заменить условием

$3^{\circ \prime}\left\|\Lambda_{1} P\left(x^{\prime}, x^{\prime \prime}, x^{\prime \prime \prime}\right)\right\| \leqslant K$ для каждого $x^{\prime}, x^{\prime \prime}, x^{\prime \prime \prime} \in S\left[x_{1} ; \max \left\{\delta ; t^{*}\right\}\right]$.

Действительно, если справедлива $3^{\circ}$, то справедлива и $3^{\circ}$ :

$$
\begin{aligned}
& \left\|E-\Lambda_{1} P\left(x^{\prime}, x^{\prime \prime}\right)\right\|=\left\|\Lambda_{1}\left[P\left(x_{1}, x_{0}\right)-P\left(x^{\prime}, x^{\prime \prime}\right)\right]\right\|= \\
= & \left\|\Lambda_{1}\left[P\left(x^{\prime}, x^{\prime \prime}\right)-P\left(x^{\prime \prime}, x_{1}\right)+P\left(x^{\prime \prime}, x_{1}\right)-P\left(x_{1}, x_{0}\right)\right]\right\|= \\
= & \left\|\Lambda_{1}\left[P\left(x^{\prime}, x^{\prime \prime}, x_{1}\right)\left(x^{\prime}-x_{1}\right)+P\left(x^{\prime \prime}, x_{1}, x_{0}\right)\left(x^{\prime \prime}-x_{0}\right)\right]\right\| \leqslant \\
\leqslant & K\left(\left\|x^{\prime}-x_{1}\right\|+\left\|x^{\prime \prime}-x_{0}\right\|\right) .
\end{aligned}
$$

Т еорем а 3 . Пусть

$1^{\circ}\left\|\Lambda_{1} P\left(x_{0}\right)\right\| \leqslant Q\left(t_{0}\right)$;

$2^{\circ}\left\|\Lambda_{1} P\left(x_{1}\right)\right\| \leqslant Q(0) ; \quad Q\left(0, t_{0}\right)=-1$;

$3^{\circ}\left\|E-\Lambda_{1} P\left(x^{\prime}, x^{\prime \prime}\right)\right\| \leqslant 1+Q\left(t^{\prime}, t^{\prime \prime}\right)$, если $x^{\prime}, x^{\prime \prime} \in S\left[x_{1}, t^{*}\right]$

u $\left\|x^{\prime}-x^{\prime \prime}\right\| \leqslant t^{\prime}-t^{\prime \prime},\left\|x^{\prime}-x_{0}\right\| \leqslant t^{\prime}-t_{0},\left\|x^{\prime \prime}-x_{1}\right\| \leqslant t^{\prime \prime} ; t^{\prime}, t^{\prime \prime} \leqslant t^{*}$;

$4^{\circ}$ уравнение (4) имеет положительное решение.

Тогда уравнение (1) имеет в сфере $S\left[x_{1} ; t^{*}\right]$ решение $x^{*}, \kappa$ которому сходится последовательность (2) со скоростью $\left\|x^{*}-x_{n}\right\| \leqslant t^{*}-t_{n}$ $(n=0,1, \ldots)$, где $t^{*}$ - наименьший положительный корень уравнения (4); $t_{n}-n$-ое приближение по методу (5) $\kappa t^{*}$.

Д ок а з а тельст в о в основном совпадает с доказательством теоремы 1. Только в данном случае не имеет место неравенство $\left\|x_{1}-x_{0}\right\| \leqslant$ $\leqslant t_{1}-t_{0}$, и поэтому возникают некоторые изменения в доказательстве неравенств $\left\|E-\Lambda_{1} P\left(x_{n}, x_{n-1}\right)\right\| \leqslant 1+Q\left(t_{n}, t_{n-1}\right) \quad(n=2,3, \ldots)$. Но последние легко установимы, так как в данном случае имеет место неравенство $\left\|x_{2}-x_{0}\right\| \leqslant t_{2}-t_{0}$. Действительно

$$
\begin{aligned}
\left\|x_{2}-x_{0}\right\| & =\left\|x_{1}-\Lambda_{1} P\left(x_{1}\right)-x_{0}\right\|=\left\|\Lambda_{1}\left[P\left(x_{1}, x_{0}\right)\left(x_{1}-x_{0}\right)-P\left(x_{1}\right)\right]\right\|= \\
& =\left\|\Lambda_{1}\left[P\left(x_{1}\right)-P\left(x_{0}\right)-P\left(x_{1}\right)\right]\right\|=\left\|\Lambda_{1} P\left(x_{0}\right)\right\| \leqslant Q\left(t_{0}\right)= \\
& =-Q\left(0, t_{0}\right)\left(-t_{0}\right)+Q(0)=Q(0)-t_{0}=t_{2}-t_{0} .
\end{aligned}
$$

Взяв в качестве мажорантного уравнения (4) уравнение

$$
Q(t) \equiv K t^{2}-\left[1+K\left(\eta_{1}-\eta_{0}\right)\right] t+\eta_{1}
$$

получим, что справедлива (как следствие из теоремы 3)

Т е о рем а 4 . Пусть

$1^{\circ}\left\|\Lambda_{1} P\left(x_{0}\right)\right\| \leqslant \eta_{0}$;

$2^{\circ}\left\|\Lambda_{1} P\left(x_{1}\right)\right\| \leqslant \eta_{1} ; \eta_{1} \leqslant \eta_{0} ;$ 
$3^{\circ}\left\|E-\Lambda_{1} P\left(x^{\prime}, x^{\prime \prime}\right)\right\| \leqslant K\left(\left\|x^{\prime}-x_{0}\right\|+\left\|x^{\prime \prime}-x_{1}\right\|\right)$ дяякаждого $x^{\prime}, x^{\prime \prime} \in S\left[x_{1}, t^{*}\right]$;

$4^{\circ} \sqrt{K}\left(\sqrt{\eta_{1}}+\sqrt{\eta_{0}}\right) \leqslant 1$.

тогда уравнение (1) имеет в сфере $S\left[x_{1} ; t^{*}\right]$ решение $x^{*}, \kappa$ которому последовательность (2) сходится. Справедливь оценки: $\left\|x^{*}-x_{n}\right\| \leqslant$ $\leqslant t^{*}-t_{n}(n=0,1, \ldots)$, гдe

$t^{*}=(2 K)^{-1}\left\{1+K\left(\eta_{1}-\eta_{0}\right)-\sqrt{\left[1+K\left(\eta_{1}-\eta_{0}\right)\right]^{2}-4 K \eta_{1}}\right\} ; \quad t_{n}-n-o e$ приближение по методу (5) $\kappa$ решению $t^{*}$ уравнения (10);

$t_{0}=\eta_{1}-\eta_{0} ; t_{1}=0$.

3 а меч ани и 2 . Если существует $P\left(x^{\prime}, x^{\prime \prime}, x^{\prime \prime \prime}\right)$, то аналогично замечанию 1 нетрудно показать, что условие $3^{\circ}$ теоремы 4 можно заменить условием

$3^{\circ \prime}\left\|\Lambda_{1} P\left(x^{\prime}, x^{\prime \prime}, x^{\prime \prime \prime}\right)\right\| \leqslant K$ для каждого

$$
x^{\prime}, x^{\prime \prime}, x^{\prime \prime \prime} \in S\left[x_{1} ; \max \left\{\left\|x_{1}-x_{0}\right\| ; i^{*}\right\}\right] \text {. }^{*}
$$

Перейдем к исследованию сходимости основного метода хорд (3). Во первых докажем теорему, опубликованную ранее автором [4] без доказательства.

Теорем а 5 . Пусть

$1^{\circ}\left\|x_{1}-x_{0}\right\| \leqslant-t_{0}$

$2^{\circ}\left\|\Lambda_{1} P\left(x_{1}\right)\right\| \leqslant Q(0) ; Q\left(0, t_{0}\right)=-1$;

$3^{\circ}\left\|\Lambda_{1}\left[P\left(x^{\prime}, x^{\prime \prime}\right)-P\left(x^{\prime \prime}, x^{\prime \prime \prime}\right)\right]\right\| \leqslant Q\left(t^{\prime}, t^{\prime \prime}\right)-Q\left(t^{\prime \prime}, t^{\prime \prime \prime}\right)$, еслu

$x^{\prime}, x^{\prime \prime}, x^{\prime \prime \prime} \in S\left[x_{0} ; t^{*}-t_{0}\right] \quad u\left\|x^{\prime}-x^{\prime \prime}\right\| \leqslant t^{\prime}-t^{\prime \prime},\left\|x^{\prime \prime}-x^{\prime \prime \prime}\right\| \leqslant t^{\prime \prime}-t^{\prime \prime \prime} ;$

$t_{0} \leqslant t^{\prime \prime \prime}, t^{\prime \prime}, t^{\prime} \leqslant t^{*}$

$4^{\circ}$ уравнение (4) имеет полонительное решение.

Тогда уравнение (1) имеет в сфере $S\left[x_{0} ; t^{*}-t_{0}\right]$ решение $x^{*}, \kappa$ которому сходится последовательность (3) со скоростью $\left\|x^{*}-x_{n}\right\| \leqslant t^{*}-t_{n}$ $(n=0,1, \ldots)$, где $t^{*}$ - наименьший положительный корень уравнения (4); $t_{n}-n$-ое приближение по методу (6) $\kappa t^{*}$.

Д оказ а тельств о. По индукции покажем сходимость последовательности (6) к $t^{*}$. Допустив, что $t_{0}<t_{1} \leqslant t_{2} \leqslant \ldots \leqslant t_{n} \leqslant t^{*}$, покажем; что $t_{n} \leqslant t_{n+1} \leqslant t^{*}$. Имеют место следующие свойства:

1) $Q(t)>0$, если $0 \leqslant t<t^{*}$.

Действительно, $Q(0)>0$ и $Q(t)$ - непрерывна

2) $Q\left(t^{*}, t^{\prime}\right) \geqslant Q\left(t^{\prime}, t^{\prime \prime}\right)$, если $0 \leqslant t^{\prime \prime} \leqslant t^{\prime} \leqslant t^{*}$.

Вытекает непосредственно из условия $3^{\circ}$.

3) $Q\left(t^{\prime}, t^{\prime \prime}\right)<0$, если $0 \leqslant t^{\prime \prime} \leqslant t^{\prime}<t^{*}$.

Действительно, $Q\left(t^{\prime}, t^{\prime \prime}\right) \leqslant Q\left(t^{*}, t^{\prime}\right)=\frac{Q\left(t^{*}\right)-Q\left(t^{\prime}\right)}{t^{*}-t^{\prime}}=-\frac{Q\left(t^{\prime}\right)}{t^{*}-t^{\prime}}<0$.

Используя эти свойства, легко получим

$$
t_{n+1}-t_{n}=-\left[Q\left(t_{n}, t_{n-1}\right)\right]^{-1} Q\left(t_{n}\right) \geqslant 0
$$

- При этом требуется симметричность оператора $P\left(x_{1}, x_{0}\right)$, т. е. $P\left(x_{1}, x_{0}\right)=P\left(x_{0}, x_{1}\right)$ 
H

$$
\begin{aligned}
t^{*}-t_{n+1} & =t^{*}-\left[Q\left(t_{n}, t_{n-1}\right)\right]^{-1} Q\left(t^{*}\right)-t_{n}+\left[Q\left(t_{n}, t_{n-1}\right)\right]^{-1} Q\left(t_{n}\right)= \\
& =t^{*}-t_{n}-\left[Q\left(t_{n}, t_{n-1}\right)\right]^{-1} Q\left(t^{*}, t_{n}\right)\left(t^{*}-t_{n}\right)= \\
& =\left[1-\frac{Q\left(t^{*}, t_{n}\right)}{Q\left(t_{n}, t_{n-1}\right)}\right]\left(t^{*}-t_{n}\right) \geqslant 0 .
\end{aligned}
$$

Итак, последовательность $\left\{t_{n}\right\}$ монотонно возрастает и ограничена. Следовательно, существует $\lim _{n \rightarrow \infty} t_{n}=\bar{t} \leqslant t^{*}$. Переходя $\mathrm{k}$ пределу в равенствах (6), получим:

$$
\bar{t}=\bar{t}-\lim _{n \rightarrow \infty} \frac{1}{Q\left(t_{n}, t_{n-1}\right)} Q(\bar{t})
$$

откуда $Q(\bar{t})=0$, т. е. $\bar{t}=t^{*}$.

Из условня $2^{\circ}$ получим

$$
\left\|x_{2}-x_{1}\right\|=\left\|\Lambda_{1} P\left(x_{1}\right)\right\| \leqslant Q(0)=t_{1}-t_{0}=-t_{0} .
$$

Поскольку по условию $3^{\circ}$

$$
\left\|\Lambda_{1}\left[P\left(x_{2}, x_{1}\right)-P\left(x_{1}, x_{0}\right)\right]\right\| \leqslant Q\left(t_{2}, 0\right)-Q\left(0, t_{0}\right)=Q\left(t_{2}, 0\right)+1<1 .
$$

то на основании теоремы Банаха существует $\Lambda_{2}=\left[P\left(x_{2}, x_{1}\right)\right]^{-1}$ ห

$$
\begin{aligned}
\left\|\Lambda_{2} P\left(x_{1}, x_{0}\right)\right\| \leqslant & \left\|\left\{E-\Lambda_{1}\left[P\left(x_{1}, x_{0}\right)-P\left(x_{2}, x_{1}\right)\right]\right\}^{-1}\right\| \leqslant \\
& \leqslant-\frac{1}{Q\left(t_{2}, 0\right)}=\frac{Q\left(0, t_{0}\right)}{Q\left(t_{2}, 0\right)} .
\end{aligned}
$$

Справедливость оценок $\left\|x_{n}-x_{n-1}\right\| \leqslant t_{n}-t_{n-1}$ и $\left\|\Lambda_{n} P\left(x_{n-1}, x_{n-2}\right)\right\| \leqslant$ $\leqslant \frac{Q\left(t_{n-1}, t_{n-2}\right)}{Q\left(t_{n}, t_{n-1}\right)}$ для любого $n$ покажем по индукции. Получим

$$
\begin{aligned}
& \left\|x_{n+1}-x_{n}\right\|=\left\|\Lambda_{n} P\left(x_{n}\right)\right\|=\| \Lambda_{n}\left\{P\left(x_{n-1}\right)+P\left(x_{n-1}, x_{n-2}\right)\left(x_{n}-x_{n-1}\right)+\right. \\
& \left.+\left[P\left(x_{n}, x_{n-1}\right)-P\left(x_{n-1}, x_{n-2}\right)\right]\left(x_{n}-x_{n-1}\right)\right\} \|= \\
& =\| \Lambda_{n} P\left(x_{n-1}, x_{n-2}\right) \Lambda_{n-1} P\left(x_{n-2}, x_{n-3}\right) \ldots \Lambda_{2} P\left(x_{1}, x_{0}\right) \Lambda_{1}\left[P\left(x_{n}, x_{n-1}\right)-\right. \\
& \left.\rightarrow P\left(x_{n-1}, x_{n-2}\right)\right]\left(x_{n}-x_{n-1}\right) \| \leqslant-\left[Q\left(t_{n}, t_{n-1}\right)\right]^{-1} Q\left(t_{n-1}, t_{n-2}\right)\left[Q\left(t_{n-1}, t_{n-2}\right)\right]^{-} \\
& \times Q\left(t_{n-2}, t_{n-3}\right) \ldots\left[Q\left(t_{2}, 0\right)\right]^{-1} Q\left(0, t_{0}\right)\left[Q\left(0, t_{0}\right)\right]^{-1}\left[Q\left(t_{n}, t_{n-1}\right)-\right. \\
& \text { - } \left.Q\left(t_{n-1}, t_{n-2}\right)\right]\left(t_{n}-t_{n-1}\right)=-\left[Q\left(t_{n}, t_{n-1}\right)\right]^{-1}\left[Q\left(t_{n}, t_{n-1}\right)-\right. \\
& \left.-Q\left(t_{n-1}, t_{n-2}\right)\right]\left(t_{n}-t_{n-1}\right)=t_{n+1}-t_{n} \text {, }
\end{aligned}
$$

и поскольку

$$
\left\|\Lambda_{n}\left[P\left(x_{n+1}, x_{n}\right)-P\left(x_{n}, x_{n-1}\right)\right]\right\|=
$$

$=\left\|\Lambda_{n} P\left(x_{n-1}, x_{n-2}\right) \Lambda_{n-1} \ldots P\left(x_{1}, x_{0}\right) \Lambda_{1}\left[P\left(x_{n+1}, x_{n}\right)-P\left(x_{n}, x_{n-1}\right)\right]\right\| \leqslant$

$$
\begin{gathered}
\leqslant-\left[Q\left(t_{n}, t_{n-1}\right)\right]^{-1} Q\left(t_{n-1}, t_{n-2}\right)\left[Q\left(t_{n-1}, t_{n-2}\right)\right]^{-1} \ldots Q\left(0, t_{0}\right)\left[Q\left(t_{n+1}, t_{n}\right)-\right. \\
\left.-Q\left(t_{n}, t_{n-1}\right)\right]=-\left[Q\left(t_{n}, t_{n-1}\right)\right]^{-1}\left[Q\left(t_{n+1}, t_{n}\right)-Q\left(t_{n}, t_{n-1}\right)\right]= \\
=1-\left[Q\left(t_{n}, t_{n-1}\right)\right]^{-1} Q\left(t_{n+1}, t_{n}\right)<1,
\end{gathered}
$$

то на основанни теоремы Банаха существует $\Lambda_{n+1}$ н 


$$
\begin{aligned}
& \left\|\Lambda_{n+1} P\left(x_{n}, x_{n-1}\right)\right\|=\left\|\left\{E-\Lambda_{n}\left[P\left(x_{n}, x_{n-1}\right)-P\left(x_{n+1}, x_{n}\right)\right]\right\}^{-1}\right\| \leqslant \\
& \leqslant \frac{1}{1-\left\{1-\left[Q\left(t_{n}, t_{n-1}\right)\right]^{-1} Q\left(t_{n+1}, t_{n}\right)\right\}}=\frac{Q\left(t_{n}, t_{n-1}\right)}{Q\left(t_{n+1}, t_{n}\right)},
\end{aligned}
$$

что и требовалось доказать.

Теперь легко получаются оценки

$$
\left\|x_{n+p}-x_{r}\right\| \leqslant t_{n+p}-t_{n},
$$

откуда при предельном переходе $(p \rightarrow \infty)$, получим

$$
\left\|x^{*}-x_{n}\right\| \leqslant t^{*}-t_{n} .
$$

Следует еще показать, что $x^{*}$ является решением уравнения (1).

Рассмотрим равенство

$$
\Lambda_{1} P\left(x_{n}\right)+\Lambda_{1} P\left(x_{n}, x_{n-1}\right)\left(x_{n+1}-x_{n}\right)=0 .
$$

Так как

$$
\begin{gathered}
\left\|\Lambda_{1} P\left(x_{n}, x_{n-1}\right)\right\|=\| \Lambda_{1}\left[P\left(x_{n}, x_{n-1}\right)-P\left(x_{n-1}, x_{n-2}\right)+\right. \\
\left.+P\left(x_{n-1}, x_{n-2}\right)-\ldots-P\left(x_{1}, x_{0}\right)+P\left(x_{1}, x_{0}\right)\right] \| \leqslant Q\left(t_{n}, t_{n-1}\right)- \\
-Q\left(t_{n-1}, t_{n-2}\right)+Q\left(t_{n-1}, t_{n-2}\right)-\ldots-Q\left(0, t_{0}\right)+1=2+Q\left(t_{n}, t_{n-1}\right)<+\infty
\end{gathered}
$$

то переходя к пределу $(n \rightarrow \infty)$ в (11) и используя непрерывность $P(x)$, получим

$$
\Lambda_{1} P\left(x^{*}\right)=0 \text { или } P\left(x^{*}\right)=0 \text {. }
$$

Теорема доказана.

Взяв в качестве мажорантного уравнения $Q(t)=0$ уравнение (9), получим, что справедлива (как следствие нз теоремы 5)

\section{Те орема 6. Пусть}

$1^{\circ} \quad\left\|x_{1}-x_{0}\right\| \leqslant \delta$

$2^{\circ} \quad\left\|\Lambda_{1} P\left(x_{1}\right)\right\| \leqslant \eta$;

$3^{\circ} \quad\left\|\Lambda_{1}\left[P\left(x^{\prime}, x^{\prime \prime}\right)-P\left(x^{\prime \prime}, x^{\prime \prime \prime}\right)\right]\right\| \leqslant K\left\|x^{\prime}-x^{\prime \prime \prime}\right\|$

для каждого

$x^{\prime}, x^{\prime \prime}, x^{\prime \prime \prime} \in S\left[x_{0}, t^{*}+\delta\right]$;

$4^{\circ} 2 \sqrt{K \eta}+K \delta \leqslant 1$.

Тогда уравнение (1) имеет в сфере $S\left[x_{0} ; t^{*}+\delta\right]$ решение $x^{*}, \kappa$ которому сходится последовательность (3) со скоростью $\left\|x^{*}-x_{n}\right\| \leqslant t^{*}-t_{n}$ $(n=0,1, \ldots)$, где $t^{*}$ - наименьший положительный корень уравнения (9); $t_{n}$ - п-ое приблинение по методу (6) $\kappa$ решению $t^{*} ; t_{0}=-\delta: t_{1}=0$.

3 а меч ан и е 3 . Если вместо условия $3^{\circ}$ теоремы 6 требовать выполнение более общего условия (ср. $[5,6])$

$$
\begin{gathered}
3^{\circ \prime} \quad\left\|\Lambda_{1}\left[P\left(x^{\prime}, x^{\prime \prime}\right)-P\left(x^{\prime \prime}, x^{\prime \prime \prime}\right)\right]\right\| \leqslant \\
\leqslant a\left\|x^{\prime}-x^{\prime \prime \prime}\right\|+b\left\|x^{\prime}-x^{\prime \prime}\right\|+b\left\|x^{\prime \prime}-x^{\prime \prime \prime}\right\|
\end{gathered}
$$

для каждого $x^{\prime}, x^{\prime \prime}, x^{\prime \prime \prime} \in S\left[x_{0} ; t^{*}+\delta\right]$, то утверждения теоремы 6 остаются справедливыми, если в них н в уравнении (9) заменить $K$ на $a+b$. 
Действительно, если $\left\|x^{\prime}-x^{\prime \prime}\right\| \leqslant t^{\prime}-t^{\prime \prime}$ и $\left\|x^{\prime \prime}-x^{\prime \prime \prime}\right\| \leqslant t^{\prime \prime}-t^{\prime \prime \prime}$, то $\left\|\Lambda_{1}\left[P\left(x^{\prime}, x^{\prime \prime}\right)-P\left(x^{\prime \prime}, x^{\prime \prime \prime}\right)\right]\right\| \leqslant a\left\|x^{\prime}-x^{\prime \prime \prime}\right\|+b\left\|x^{\prime}-x^{\prime \prime}\right\|+b\left\|x^{\prime \prime}-x^{\prime \prime \prime}\right\| \leqslant$ $\leqslant a\left(t^{\prime}-t^{\prime \prime \prime}\right)+b\left(t^{\prime}-t^{\prime \prime}\right)+b\left(t^{\prime \prime}-t^{\prime \prime \prime}\right)=(a+b)\left(t^{\prime}-t^{\prime \prime \prime}\right)$

и следовательно можно выбрать

$$
Q(t)=(a+b) t^{2}-[1-(a+b) \delta] t+\eta .
$$

В этом виде теорема 6 уточняет по существу результат И. В. Шмидта $[5,6]$.

Аналогично тому, как теорема 3 получена из теоремы 1 , из теоремы 5 получается

Те орема 7 . Пусть

$1^{\circ} \quad\left\|\Lambda_{1} P\left(x_{0}\right)\right\| \leqslant Q\left(t_{0}\right) ; \quad Q\left(0, t_{0}\right)=-1 ;$

$2^{\circ} \quad\left\|\Lambda_{1} P\left(x_{1}\right)\right\| \leqslant Q(0)$;

$3^{\circ}\left\|\Lambda_{1}\left[P\left(x^{\prime}, x^{\prime \prime}\right)-P\left(x^{\prime \prime}, x^{\prime \prime \prime}\right)\right]\right\| \leqslant Q\left(t^{\prime}, t^{\prime \prime}\right)-Q\left(t^{\prime \prime}, t^{\prime \prime \prime}\right)$, eсли $x^{\prime}, x^{\prime \prime}, x^{\prime \prime \prime} \in$ $6 S\left[x_{1} ; \max \left\{\left\|x_{1}-x_{0}\right\| ; t^{*}\right\}\right]$ и $\left\|x^{\prime}-x^{\prime \prime}\right\| \leqslant t^{\prime}-t^{\prime \prime},\left\|x^{\prime}-x^{\prime \prime \prime}\right\| \leqslant t^{\prime}-t^{\prime \prime \prime}$; $t_{0} \leqslant t^{\prime \prime \prime}, t^{\prime \prime}, t^{\prime} \leqslant t^{*}$

$4^{\circ}$ уравнение (4) имеет положительное решение.

Тогда уравнение (1) имеет в сфере $S\left[x_{1} ; \max \left\{\left\|x_{1}-x_{0}\right\| ; t^{*}\right\}\right]$ решение $x^{*}, \kappa$ которому сходится последовательность (3) со скоростью $\left\|x^{*}-x_{n}\right\| \leqslant t^{*}-t_{n}(n=0,1, \ldots)$, где $t^{*}-$ наименьший положительньй корень уравнения (4); $t_{n}-n$-ое приближение по методу (6) $\kappa t^{*}$.

Взяв в качестве мажорантного уравнения $Q(t)=0$ уравнение $(10)$. получим, что справедлива (как следствие из теоремы 7)

\section{Те орем а 8 . Пусть}

$1^{\circ} \quad\left\|\Lambda_{1} P\left(x_{0}\right)\right\| \leqslant \eta_{0}$;

$2^{\circ} \quad\left\|\Lambda_{1} P\left(x_{1}\right)\right\| \leqslant \eta_{1} ; \eta_{1} \leqslant \eta_{0} ;$

$3^{\circ} \quad\left\|\Lambda_{1}\left[P\left(x^{\prime}, x^{\prime \prime}\right)-P\left(x^{\prime \prime}, x^{\prime \prime \prime}\right)\right]\right\| \leqslant K\left\|x^{\prime}-x^{\prime \prime \prime}\right\|$ для каждого

$x^{\prime}, x^{\prime \prime}, x^{\prime \prime \prime} \in S\left[x_{1} ; \max \left\{\left\|x_{1}-x_{0}\right\| ; t^{*}\right\}\right]$;

$4^{0} \quad \sqrt{K}\left(\sqrt{\eta_{1}}+\sqrt{\eta_{0}}\right) \leqslant 1$.

Тогда уравнение (1) имеет в сфере $S\left[x_{0} ; t^{*}+\eta_{0}-\eta_{1}\right]$ решение $x^{*}, \kappa$ которому сходится последовательность (3) со скоростью $\left\|x^{*}-x_{n}\right\| \leqslant$ $\leqslant t^{*}-t_{n}(n=0,1, \ldots)$, где $t^{*}$ - наименьший положительный корень уравнения (10); $t_{n}$ - n-ое приближение по методу (6) ' $\kappa$ решению $t$ *; $t_{0}=\eta_{1}-\eta_{0} ; t_{1}=0$.

3 а м е ч а и е 4. Теоремы 6 и 8 остаются справедливыми, если в них условие $3^{\circ}$ заменить условием

$\left\|\Lambda_{1} P\left(x^{\prime}, x^{\prime \prime}, x^{\prime \prime \prime}\right)\right\| \leqslant K$ для каждого $x^{\prime}, x^{\prime \prime}, x^{\prime \prime \prime}$ соответственно в сферах $S\left[x_{0} ; t^{*}+\delta\right]$ и $S\left[x_{1} ; \max \left\{\left\|x_{1}-x_{0}\right\| ; t^{*}\right\}\right]$.

Как уже отмечено автором ранее $\left[^{4}\right]$, теорема 8 уточняет результаты А. С. Сергеева [3].

Докажем еще две теоремы о сходимости метода (2) в предположении, что известны существование и область расположения решения $x^{*}$ уравнения (1). 
Т е о рем а 9 . Пусть

$1^{\circ}\left\|x^{*}-x_{1}\right\| \leqslant t^{*}-t_{1}$

$2^{\circ}\left\|E-\Lambda_{1} P\left(x^{*}, x\right)\right\| \leqslant 1+Q\left(t^{*}, t\right)$, если $\left\|x^{*}-x\right\| \leqslant t^{*}-t \leqslant t^{*}-t_{1}$;

$3^{\circ}$ уравнение (4) не имеет решении в промежутке $t_{1}<t<t^{*}$;

$4^{\circ} Q\left(t_{1}\right)>0$.

Тогда последовательность (2) сходится $\kappa$ решению $x^{*}$ уравнения (1). причем $\left\|x^{*}-x_{n}\right\| \leqslant t^{*}-t_{n}(n=1,2, \ldots)$, где $t^{*}$ - наименьшее положительное решение уравнения (4): $t_{n}$ - n-ое приближение $\kappa$ решению $t^{*}$ по методу $(5) ; t_{1}=0$.

Дока з а тельств о. По условию $1^{\circ}\left\|x^{*}-x_{1}\right\| \leqslant t^{*}-t_{1}=t^{*}$. Для доказательетва неравенств $\left\|x^{*}-x_{n}\right\| \leqslant t^{*}-t_{n}$ для любого $n$ используем принцип полной индукции

$$
\begin{gathered}
\left\|x^{*}-x_{n}\right\|=\left\|x^{*}-x_{n-1}+\Lambda_{1} P\left(x_{n-1}\right)-\Lambda_{1} P\left(x^{*}\right)\right\|= \\
=\left\|\left[E-\Lambda_{1} P\left(x^{*}, x_{n-1}\right)\right]\left(x^{*}-x_{n-1}\right)\right\| \leqslant \\
\leqslant\left[1+Q\left(t^{*}, t_{n-1}\right)\right]\left(t^{*}-t_{n-1}\right)=t^{*}-t_{n-1}-Q\left(t_{n-1}\right)=t^{*}-t_{n} .
\end{gathered}
$$

На основании полученных оценок последовательность $\left\{t_{n}\right\}$ ограничена $\left(\leqslant t^{*}\right)$. Так как по условиям $3^{\circ}$ и $4^{\circ}$ функция $Q(t)$ положительна в промежутке $t_{1} \leqslant t<t^{*}$, то $t_{n+1}=t_{n}+Q\left(t_{n}\right) \geqslant t_{n}$, т. е. $\left\{t_{n}\right\}$ является и монотонно возрастающей. Итак, существует $\lim t_{n}=t^{*}$. Переходя к пределу $(n \rightarrow \infty)$ в (4), получим $\bar{t}=\bar{t}+Q(\bar{t})$ или $Q(\bar{t})=0$ или $\bar{t}=t^{*}$. Следовательно $\lim x_{n}=x^{*}$. Теорема доказана.

Взяв в качестве мажорантного уравнения $Q(t)=0$ уравнение

$$
Q(t)=\left[1-K_{2}\left(2 t^{*}-t+\delta\right)-K_{3} t^{*}\right]\left(t^{*}-t\right)
$$

получим, что справедлива (как следствие из теоремы 9)

Т е о рем а 10. Пусть

$1^{\circ}$ уравнение (1) имеет решение в сфере $\left\|x^{*}-x_{1}\right\| \leqslant t^{*}$;

$2^{\circ}\left\|x_{1}-x_{0}\right\| \leqslant \delta$;

$3^{\circ}$ a) $\left\|E-\Lambda_{1} P\left(x, x_{1}\right)\right\| \leqslant K_{1}\left\|x-x_{0}\right\|$, ecлu $x \in S\left[x_{1}, t^{*}\right]$;

б) $\left\|E-\Lambda_{1} P\left(x^{\prime}, x^{\prime \prime}\right)\right\| \leqslant K_{2}\left\|x^{\prime \prime}-x_{0}\right\|+K_{3}\left\|x^{\prime}-x_{1}\right\|$, ecли $x^{\prime} \in S\left[x_{1}, t^{*}\right]$ $u x^{\prime \prime} \in S\left[x_{1}, 2 t^{*}-t_{2}\right]$

$4^{\circ} K_{2}\left(1+K_{1} t^{*}\right)\left(t^{*}+\delta\right)+K_{3} t^{*}<1$.

Тогда последовательность (2) сходится $\kappa$ единственному в сфере $\left\|x-x_{1}\right\| \leqslant t^{*}$ решению $x^{*}$ уравнения (1). Справедливы оценки: $\left\|x^{*}-x_{2}\right\| \leqslant K_{1}\left(t^{*}+\delta\right) t^{*} ; \quad\left\|x^{*}-x_{n}\right\| \leqslant t^{*}-t_{n} \quad(n=3,4, \ldots), \quad$ где $t_{n+1}=t_{n}+Q\left(t_{n}\right)(n=2,3, \ldots) ; t_{2}=\left[1-K_{1}\left(t^{*}+\delta\right)\right] t^{*}$.

Доказ ательство. Так как

$$
x^{*}-x_{2}=x^{*}-x_{1}+\Lambda_{1} P\left(x_{1}\right)-\Lambda_{1} P\left(x^{*}\right)=\left[E-\Lambda_{1} P\left(x^{*}, x_{1}\right)\right]\left(x^{*}-x_{1}\right),
$$

To

$$
\left\|x^{*}-x_{2}\right\| \leqslant K_{1}\left(t^{*}+\delta\right) t^{*}=t^{*}-t_{2}
$$


Теперь применим теорему 9, рассмотрев в (2) и (5) в качестве начальных приближений соответственно $x_{2}$ и $t_{2}$. Так как сфера $\left\|x^{*}-x\right\| \leqslant$ $\leqslant t^{*}-t_{2}$ содержится в сфере $\left\|x-x_{1}\right\| \leqslant 2 t^{*}-t_{2}$, то при $\left\|x^{*}-x\right\| \leqslant$ $\leqslant t^{*}-t$

$$
\begin{aligned}
& \left\|E-\Lambda_{1} P\left(x^{*}, x\right)\right\| \leqslant K_{2}\left\|x-x_{0}\right\|+K_{3}\left\|x^{*}-x_{1}\right\| \leqslant \\
& \leqslant K_{2}\left(\left\|x-x^{*}\right\|+\left\|x^{*}-x_{0}\right\|\right)+K_{3}\left\|x^{*}-x_{1}\right\| \leqslant \\
& \leqslant K_{2}\left(2 t^{*}-t+\delta\right)+K_{3} t^{*}=1+Q\left(t^{*}, t\right)
\end{aligned}
$$

т. е. условие $2^{\circ}$ теоремы 9 выполнено. Условие $4^{\circ}$ данной теоремы соответствует условию $Q\left(t_{2}\right)>0$. Уравнение (13) не имее решений в промежутке $t_{2}<t<t^{*}$.

Итак, все условия теоремы 9 выполнены и следовательно $\lim x_{n}=x^{*}$. Легко видеть, что решение $x^{*}$ является единственным в сфере $\left\|x-x_{1}\right\| \leqslant t^{*}$.

Теорема доказана.

3 а меч ан ие 5 . Из теорем $2,4,6,8,10$ вытекает по сушеству в предельном случае $\left(x_{1}=x_{0}\right.$, т. е. $\delta=0$ и $\eta_{1}=\eta_{0}=\eta ; \quad P(x, x, x)=$ $=\frac{1}{2} P^{\prime \prime}(x)$, т. е. если $K, K_{1}, K_{2}, K_{3}$ заменить $\left.\frac{1}{2} K\right)$ основные утверждения теорем о сходимости метода Ньютона (ср. [1, 2]). Итак, вышеизложенные теоремы можно рассматривать как обобщения теорем Л. В. Канторовича и И. П. Мысовских о сходимости метода Ньютона, который является предельным случаем метода хорд.

Л Н Т Е Р A T Y P A

1. Канторович Л. Н., Вестник ЛГУ, 2, № 7, 68-103 (1957).

2. Мысковских И. П., Вестник ЛГУ, № 11, 25-48 (1953).

3. Се ргее в А. С., Сибирский матем. журнал, 2, № 2, 282-289 (1961).

4. у ль м С., Изв. АН ЭССР, Сер. физ.-матен. и техн. наук, 12, № 1, 24-30 (1963).

5. Schmidt J. W., Z. angew. Math. und Mech., 41, Sonderheft, 61-63 (1961).

6. Schmidt J. W., Z. angew. Math. und Mech., 43, Heft 1/2, $1-8$ (1963).

Ннститут кибернетики

Академии наук Эстонской ССР
Поступила в редакцию 27. II 1964

\title{
MAJORANTIDE PRINTSIIP JA KÖOLLUDE MEETOD
}

\author{
S. Uim, \\ füüsika-matemaatikateaduste kandidaat
}

Resümee

Majorantide printsiibi [ $\left.{ }^{1}\right]$ abil tõestatakse rida teoreeme kõölude meetodi $(2,3)$ koonduvusest lineaarses normeeritud ruumis. Teoreemid 6 ja 8 täpsustavad vastavalt J. W. Schmiditi [5,6] ja A. S. Sergejevi [3] tulemusi. Töestatud teoreeme võib ühtlasi vaadelda Newtoni meetodi koonduvusteoreemide $\left[{ }^{1,2}\right]$ üldistustena.

Eesti NSV Teaduste Akadeemia Küberneetika Instituut
Saabus toimetusse 27. II 1964 


\section{THE PRINCIPLE OF MAJORANTS AND THE SECANT METHOD}

\section{S. Uim}

\section{Summary}

Using the principle of majorants, some theorems on the convergence of the secant method are proved in the linear normed spaces. The results of J. W. Schmidt $[5,6]$ and A. S. Sergeyev [3] are made more precise by theorems 6 and 8 respectively. The proved theorems may also be considered as generalizations of the theorems on the convergence of Newton's method $\left[{ }^{1,2}\right]$.

\section{Academy of Sciences of the Estonian S.S.R.,} Institute of Cybernetics

Received

Feb. 27th, 1964 